Miroslav Repický, Mathematical Institute of Slovak Academy of Sciences, Jesenná 5, 04154 Košice, Slovakia, e-mail: repicky@@kosice.upjs.sk

\title{
TOWERS AND PERMITTED TRIGONOMETRIC THIN SETS
}

\begin{abstract}
In [3] we introduced the notion of perfect measure zero sets and proved that every perfect measure zero set is permitted for any of the four families of trigonometric thin sets $\mathcal{N}, \mathcal{A}, \mathcal{N}_{0}$, and $p \mathcal{D}$. Now we prove that the unions of less than $\mathfrak{t}$ perfect measure zero sets are permitted for the mentioned families. This strengthens a result of T. Bartoszyński and M. Scheepers [1] saying that every set of cardinality less than $\mathfrak{t}$ is $\mathcal{N}$-permitted.
\end{abstract}

\section{Introduction}

Let $\mathcal{F}$ be a family of sets of reals. Let $A, B \in \mathcal{F}$. We say that a set $A$ is permitted for $\mathcal{F}$ if $A \cup B \in \mathcal{F}$ for every $B \in \mathcal{F}$. Let $A$ be a set of reals. Then $A$ is a $p D$-set (pseudo Dirichlet set) if there is an increasing sequence of integers $\left\{n_{k}\right\}_{k=0}^{\infty}$ such that the sequence $\left\{\sin n_{k} \pi x\right\}_{k=0}^{\infty}$ converges quasinormally on $A$; i.e. there is a sequence of positive reals $\left\{\varepsilon_{k}\right\}_{k=0}^{\infty}$ converging to 0 such that $(\forall x \in A)\left(\forall^{\infty} k\right)\left|\sin n_{k} \pi x\right|<\varepsilon_{k}$. A is an $\mathrm{N}_{0}$-set if there is an increasing sequence of integers $\left\{n_{k}\right\}_{k=0}^{\infty}$ such that $\sum_{k=0}^{\infty}\left|\sin n_{k} \pi x\right|<\infty$ for $x \in A$. $A$ is an $A$-set if there is an increasing sequence of integers $\left\{n_{k}\right\}_{k=0}^{\infty}$ such that $\left\{\sin n_{k} \pi x\right\}_{k=0}^{\infty}$ converges to 0 for $x \in A$. $A$ is an $N$-set if there is a sequence of non-negative reals $\left\{\rho_{n}\right\}_{n=0}^{\infty}$ such that $\sum_{n=0}^{\infty} \rho_{n}=\infty$ and the series $\sum_{n=0}^{\infty}\left|\rho_{n} \sin n \pi x\right|$ converges for $x \in A$. The families of all $p D$-sets, $N_{0}$-sets, $A$-sets, and $N$-sets are denoted by $p \mathcal{D}, \mathcal{N}_{0}, \mathcal{A}$, and $\mathcal{N}$, respectively.

Key Words: $N$-sets, $A$-sets, $N_{0}$-sets, pseudo Dirichlet sets, permitted sets, perfect measure zero sets

Mathematical Reviews subject classification: Primary: 42A20. Secondary: 03E05, $03 \mathrm{E} 20$

Received by the editors October 12, 1995

*The work has been supported by grant 2/1224/95 of Slovenská grantová agentúra. 
A set $A$ has perfect measure zero if for every sequence of positive reals $\left\{\varepsilon_{n}\right\}_{n=1}^{\infty}$ there is an increasing sequence of integers $\left\{n_{k}\right\}_{k=0}^{\infty}$ and a sequence of finite families of intervals $\left\{\mathcal{I}_{n}\right\}_{n=1}^{\infty}$ such that $\left|\mathcal{I}_{n}\right| \leq n,|I|<\varepsilon_{n}$ for every $I \in \mathcal{I}_{n}$ and $A \subseteq \bigcup_{m} \bigcap_{k>m} \bigcup \mathcal{I}_{n_{k}}$.

In [3] it was proved that every perfect measure zero set is permitted for any of the families $\mathcal{N}, \mathcal{A}, \mathcal{N}_{0}$, and $p \mathcal{D}$; every $\gamma$-set has perfect measure zero; every perfect measure zero set has strong measure zero; the subgroup of $\mathbb{R},+$ generated by a set having perfect measure zero has perfect measure zero; and every set of cardinality less than the additivity of Lebesgue measure has perfect measure zero.

A result of [1] says that every set of cardinality less than $\mathfrak{t}$ is permitted for $\mathcal{N}$. The cardinals $t$ and the additivity of Lebesgue measure are two the best known lower bounds for the minimal size of a set not being permitted for $\mathcal{N}$. It is worth mentioning that these two bounds are mutually independent. (See a discussion in [3].) Some more bounds of cardinal invariants of other families of trigonometric thin sets can be found in [2] and [1]. Using some ideas of [1] we prove the following result.

Main Theorem 1.1 Let $\mathcal{F}$ be any of the families $\mathcal{N}, \mathcal{A}, \mathcal{N}_{0}$ and $p \mathcal{D}$. The unions of less than $\mathfrak{t}$ sets having perfect measure zero are permitted for $\mathcal{F}$.

Let us recall that $\mathfrak{t}$ is the minimal size of a tower of subsets of $\omega, \mathfrak{h}$ is the distributivity number of the Boolean algebra $\mathcal{P}(\omega) / f i n$, and $\mathfrak{b}$ is the minimal size of an unbounded family of functions under the eventual dominance. It is well known that $\mathfrak{t} \leq \mathfrak{h} \leq \mathfrak{b}$.

\section{The Proof of the Case $\mathcal{N}$}

Let $E$ be an $N$-set, i.e. there is a sequence $\left\{\rho_{n}\right\}_{n=1}^{\infty}$ such that $\sum_{n=1}^{\infty} \rho_{n}=\infty$ and $E=\left\{x \in \mathbb{R}: \sum_{n=1}^{\infty} \rho_{n}|\sin n \pi x|<\infty\right\}$. Let us denote $s_{n}=\sum_{k=1}^{n} \rho_{k}$. There is a surjective monotone function $\iota: \omega \rightarrow \omega \backslash\{0\}$ such that

$$
\sum_{n=1}^{\infty} \frac{\rho_{n}}{s_{n}^{1+1 / \iota^{2}(n)}}<\infty
$$

Let $\rho_{n}^{\prime}=\rho_{n} / s_{n}$ and $\delta_{n}=s_{n}^{-1 / \iota^{2}(n)}$. Then $\sum_{n=1}^{\infty} \rho_{n}^{\prime}=\infty$ and $\sum_{n=1}^{\infty} \rho_{n}^{\prime} \delta_{n}<\infty$. There is an increasing function $f \in \omega^{\omega} \omega$ such that $\sum_{n=f(k)}^{f(k+1)-1} \rho_{n}^{\prime} \geq 1$ for all $k \in \omega$. Let us denote $J_{k}=[f(k), f(k+1))$ and

$$
\varepsilon_{m}=\min \left\{\frac{\delta_{n}}{n s_{n}}:(\exists k) \iota(k)=m \& n \in J_{k}\right\} .
$$


Lemma 2.1 Suppose $Z \subseteq \omega$ is a finite set, $0<\delta \leq 1$ and $x_{1}, \ldots, x_{n} \in \mathbb{R}$. There is $Z^{\prime} \subseteq Z$ such that $\left|Z^{\prime}\right| \geq \delta^{n}|Z|$ and

$$
\left(\forall i, j \in Z^{\prime}\right)(\forall l=1, \ldots, n)\left|\sin (i-j) \pi x_{l}\right|<2 \pi \delta .
$$

Proof. First let $n=1$. Find $m$ such that $1 /(2 m)<\delta \leq 1 / m$ and divide the interval $[0,1]$ into $m$ subintervals of length $1 / m$. There is a set $Z^{\prime} \subseteq Z$ such that $\left|Z^{\prime}\right| \geq|Z| / m \geq \delta|Z|$ and for all $i \in Z^{\prime},\left\{i x_{1}\right\}$ are in the same subinterval. (Let us recall that $\{y\}$ denotes the fractional part of a real $y$.) Hence $\left|\sin (i-j) \pi x_{1}\right| \leq \pi / m<2 \pi \delta$ for all $i, j \in Z^{\prime}$. Now in case $n>1$ apply the previous result $n$ times.

Lemma 2.2 There is a system of functions $f_{\alpha} \in{ }^{\omega} \mathbb{R}$ for $\alpha<\mathfrak{b}$ such that

(1) $\left(\forall^{\infty} n\right) 0 \leq f_{\alpha}(n) \leq s_{n} \delta_{n}^{\iota(n)}$,

(2) $(\forall k \in \omega) \lim _{n \rightarrow \infty} f_{\alpha}(n) \delta_{n}^{k \iota(n)}=\infty$,

(3) If $\beta<\alpha<\mathfrak{b}$, then $\left(\forall^{\infty} n\right) f_{\alpha}(n) \leq f_{\beta}(n) \delta_{n}^{\iota(n)}$.

Proof. Let us set $f_{0}(n)=s_{n} \delta_{n}^{\iota(n)}$ and similarly in the non-limit steps let us set $f_{\alpha+1}(n)=f_{\alpha}(n) \delta_{n}^{\iota(n)}$. Then we get

$$
\lim _{n \rightarrow \infty} f_{0}(n) \delta_{n}^{k \iota(n)}=\lim _{n \rightarrow \infty} s_{n}^{1-(k+1) / \iota(n)} \geq \lim _{n \rightarrow \infty} s_{n}^{1 / 2}=\infty
$$

and condition (2) can be easily verified also for the non-limit step. Let $\alpha<\mathfrak{b}$ be limit. For each $\beta<\alpha$ there is $g_{\beta} \in \omega^{\omega} \omega$ with $\lim _{n \rightarrow \infty} g_{\beta}(n)=\infty$ such that $\lim _{n \rightarrow \infty} f_{\beta}(n) \delta_{n}^{g_{\beta}(n) \iota(n)}=\infty$ (by (2) in the induction hypothesis). Let $g \in{ }^{\omega} \omega$ be such that $\lim _{n \rightarrow \infty} g(n)=\infty$ and $(\forall \beta<\alpha)\left(\forall^{\infty} n\right) g(n) \leq g_{\beta}(n)$. Then for any $\beta<\alpha, \lim _{n \rightarrow \infty} f_{\beta}(n) \delta_{n}^{g(n) \iota(n)}=\infty$. We can find $h \in \omega_{\omega}^{\omega}$ with $\lim _{n \rightarrow \infty} h(n)=\infty$ such that $(\forall \beta<\alpha)\left(\forall^{\infty} n\right) h(n) \leq f_{\beta}(n) \delta_{n}^{g(n) \iota(n)}$. Let us set $f_{\alpha}(n)=h(n) \delta_{n}^{-g(n) \iota(n)}$. Then

$$
\lim _{n \rightarrow \infty} f_{\alpha}(n) \delta_{n}^{k \iota(n)}=\lim _{n \rightarrow \infty} h(n) \delta_{n}^{(k-g(n)) \iota(n)} \geq \lim _{n \rightarrow \infty} h(n)=\infty
$$

for all $k \in \omega$ and

$$
\left(\forall^{\infty} n\right) f_{\alpha}(n)=h(n) \delta_{n}^{-g(n) \iota(n)} \leq f_{\beta+1}(n) \leq f_{\beta}(n) \delta_{n}^{\iota(n)}
$$

for all $\beta<\alpha$.

Lemma 2.3 Let $\kappa<\mathfrak{h}$, let $A_{\alpha}, \alpha<\kappa$, be perfectly measure zero sets, let $a$ be an infinite subset of $\omega$ and let $\left\{\varepsilon_{n}\right\}_{n=1}^{\infty}$ be a given sequence of positive 
reals. There is a sequence of integers $\left\{n_{k}\right\}_{k=0}^{\infty}$ elements of a and a system of sequences of finite systems of intervals $\left\{\mathcal{I}_{n}^{\alpha}\right\}_{n=1}^{\infty}, \alpha<\kappa$, such that $\left|\mathcal{I}_{n}^{\alpha}\right| \leq n$, $\left(\forall I \in \mathcal{I}_{n}^{\alpha}\right)|I|<\varepsilon_{n}$ and $A_{\alpha} \subseteq \bigcup_{m} \bigcap_{k \geq m} \bigcup \mathcal{I}_{n_{k}}^{\alpha}$ for each $\alpha$. (The sequence $\left\{n_{k}\right\}_{k=0}^{\infty}$ is independent of $\alpha$.)

Proof. Let $\mathfrak{I}$ be the family of all infinite sequences of finite families of intervals $\left\{\mathcal{I}_{n}\right\}_{n=1}^{\infty}$ such that $\left|\mathcal{I}_{n}\right| \leq n$ and $\left(\forall I \in \mathcal{I}_{n}\right)|I|<\varepsilon_{n}$. The sets

$$
D_{\alpha}=\left\{b \in[\omega]^{\omega}:\left(\exists\left\{\mathcal{I}_{n}\right\}_{n=1}^{\infty} \in \mathfrak{I}\right) A_{\alpha} \subseteq \bigcup_{m} \bigcap_{n \in b \backslash m} \bigcup \mathcal{I}_{n}\right\}
$$

are open dense in $[\omega]^{\omega}$. (See the proof of Theorem 1.2 (iii) of [3].) Choose $b \subseteq a, b \in \bigcap_{\alpha<\kappa} D_{\alpha}$ and let $\left\{n_{k}\right\}_{k=0}^{\infty}$ be the increasing enumeration of the set $b$.

The last lemma enables us to choose $n_{k}$ uniformly for a given family of perfect measure zero sets and so for the case of $N$-sets in the Main Theorem it is enough to prove the following proposition. Notice that in the proposition $\iota\left(n_{k}\right)$ can be replaced by $n_{k}$. (But it is easier handling terms $\iota\left(n_{k}\right)$ in the proof.)

Proposition 2.4 Let $\left\{n_{k}\right\}_{k=0}^{\infty}$ be a given increasing sequence of integers and $\left\{\varepsilon_{n}\right\}_{n=1}^{\infty}$ be the sequence of positive reals defined by (2.1) for a given $N$-set $E$. Whenever $\nu<\mathfrak{t}$ and $\left\{\mathcal{I}_{n}^{\alpha}\right\}_{n=1}^{\infty}, \alpha \leq \nu$, are sequences of finite families of intervals such that $\left|\mathcal{I}_{n}\right| \leq n$ and $\left(\forall I \in \mathcal{I}_{n}\right)|I| \leq \varepsilon_{n}$, then $E \cup$ $\bigcup_{\alpha \leq \nu} \bigcup_{m} \bigcap_{k \geq m} \cup \mathcal{I}_{\iota\left(n_{k}\right)}^{\alpha}$ is an $N$-set.

ProOF. Let $\mathcal{I}_{n}^{\alpha}=\left\{\left[a_{n, l}^{\alpha}, a_{n, l}^{\alpha}+\varepsilon_{n}\right]\right\}_{l=1}^{n}$ for $n \geq 1$ and $\alpha \leq \nu$ and let us set $P_{\alpha}=\bigcup_{m} \bigcap_{k \geq m} \bigcup \mathcal{I}_{\iota\left(n_{k}\right)}^{\alpha}$. By induction we build $\left\{\varphi_{\alpha}: \alpha \leq \nu\right\}$ and $\left\{a_{\alpha}: \alpha \leq\right.$ $\nu\} \subseteq[\omega]^{\omega}$ such that

(1) $\varphi_{\alpha}: \bigcup_{k \in a_{\alpha}} J_{n_{k}} \rightarrow[\omega]^{<\omega}$

(2) $\left(\forall k \in a_{\alpha}\right)\left(\forall n \in J_{n_{k}}\right)\left(\forall i, j \in \varphi_{\alpha}(n)\right)\left(\forall l=1, \ldots, \iota\left(n_{k}\right)\right)$

$\left|\sin (i-j) n \pi a_{\iota\left(n_{k}\right), l}^{\alpha}\right|<2 \pi \delta_{n}$,

(3) $\left(\forall n \in \operatorname{dom}\left(\varphi_{\alpha}\right)\right) \max \varphi_{\alpha}(n) \leq s_{n}$,

(4) $\left(\forall^{\infty} n \in \operatorname{dom}\left(\varphi_{\alpha}\right)\right)\left|\varphi_{\alpha}(n)\right| \geq f_{\alpha}(n)$,

(5) If $\beta \leq \alpha$, then $a_{\alpha} \subseteq^{*} a_{\beta}$ and $\left(\forall^{\infty} n \in \operatorname{dom}\left(\varphi_{\alpha}\right)\right) \varphi_{\alpha}(n) \subseteq \varphi_{\beta}(n)$.

For $\alpha=0$ set $a_{0}=\omega$ and for $n \in J_{n_{k}}$. Using Lemma 2.1 find $\varphi_{0}(n) \subseteq\{i$ : $\left.i \leq s_{n}\right\}$ so that $\left|\varphi_{0}(n)\right| \geq s_{n} \delta_{n}^{\iota\left(n_{k}\right)} \geq s_{n} \delta_{n}^{\iota(n)} \geq f_{0}(n)$ and

$$
\left(\forall i, j \in \varphi_{0}(n)\right)\left|\sin (i-j) n \pi a_{\iota\left(n_{k}\right), l}^{0}\right|<2 \pi \delta_{n}
$$


(whenever $\delta_{n} \leq 1$ ). Similarly using Lemma 2.1 we can find $\varphi_{\alpha+1}(n) \subseteq \varphi_{\alpha}(n)$ so that (2) and (4) hold and we can set $a_{\alpha+1}=a_{\alpha}$. Let $\alpha \leq \nu$ be limit. For $k \in \omega$ let

$$
\begin{aligned}
U_{k}= & \left\{F \in \in^{J_{n_{k}}}\left([\omega]^{<\omega}\right):(\forall n \in \operatorname{dom}(F)) \max F(n) \leq s_{n}\right. \\
& \&|F(n)| \geq f_{\alpha}(n) \&(\forall i, j \in F(n))\left(\forall l=1, \ldots, \iota\left(n_{k}\right)\right) \\
& \left.\left|\sin (i-j) \pi n a_{\iota\left(n_{k}\right), l}^{\alpha}\right|<2 \pi \delta_{n}\right\} .
\end{aligned}
$$

The set $U=\bigcup_{k \in \omega} U_{k}$ is countably infinite. For $\beta<\alpha$ let

$$
X_{\beta}=\left\{F \in U:(\forall n \in \operatorname{dom}(F)) F(n) \subseteq \varphi_{\beta}(n)\right\} .
$$

For all $n \in J_{n_{k}}, \iota(n) \geq \iota\left(n_{k}\right)$. Hence for all but finitely many $k$ for all $n \in J_{n_{k}}$, $f_{\alpha}(n) \leq f_{\beta}(n) \delta_{n}^{\iota(n)} \leq f_{\beta}(n) \delta_{n}^{\iota\left(n_{k}\right)}$ and using Lemma 2.1 we easily see that $X_{\beta}$ is infinite. Moreover, whenever $\beta<\gamma<\alpha$, then $X_{\gamma} \subseteq^{*} X_{\beta}$. Since $\alpha<\mathfrak{t}$, there is $X \subseteq U$ such that $X \subseteq^{*} X_{\beta}$ for all $\beta<\alpha$. Since each $U_{k}$ is finite we can choose $X$ so that $\left|X \cap U_{k}\right| \leq 1$ for each $k \in \omega$. Let us define $\varphi_{\alpha}=\bigcup X$ and $a_{\alpha}=\left\{k: X \cap U_{k} \neq \emptyset\right\}$.

Let $\varphi=\varphi_{\nu}$ and let $a \subseteq \omega$ be the set of all $k \in a_{\nu}$ such that for each $n \in J_{n_{k}}$ there are $i_{n}, j_{n} \in \varphi(n), j_{n}<i_{n}$, and for each such $n$ let us put $\lambda_{n}=i_{n}-j_{n}$; $\lambda_{n} \leq s_{n}$. The set $a$ contains all but finitely many $k \in a_{\nu}$. We prove that the series

$$
\sum_{k \in a} \sum_{n \in J_{n_{k}}} \rho_{n}^{\prime}\left|\sin \lambda_{n} n \pi x\right|
$$

converges for $x \in E \cup \bigcup_{\alpha \leq \nu} P_{\alpha}$. This finishes the proof since

$$
\sum_{k \in a} \sum_{n \in J_{n_{k}}} \rho_{n}^{\prime}=\infty
$$

First notice that by (2) for all but finitely many $k \in a$ and for all $n \in J_{n_{k}}$

$$
\left|\sin \lambda_{n} n \pi a_{\iota\left(n_{k}\right), l}^{\alpha}\right| \leq 2 \pi \delta_{n} \quad \text { for } l=1, \ldots, \iota\left(n_{k}\right)
$$

and since

$$
\left|\sin \lambda_{n} n \pi \varepsilon_{\iota\left(n_{k}\right)}\right| \leq \lambda_{n} n \varepsilon_{\iota\left(n_{k}\right)} \leq s_{n} n \varepsilon_{\iota\left(n_{k}\right)} \leq \delta_{n}
$$

we obtain that

$$
\left|\sin \lambda_{n} n \pi x\right| \leq 3 \pi \delta_{n} \quad \text { for } x \in \bigcup \mathcal{I}_{\iota\left(n_{k}\right)}^{\alpha} \text { and } n \in J_{n_{k}}
$$

holds for all but finitely many $k \in a$. 
Let $x \in P_{\alpha}, \alpha \leq \nu$. There is $m$ such that $x \in \bigcup^{\mathcal{I}_{\iota\left(n_{k}\right)}^{\alpha}}$ and (2.2) holds for all $k \in a \backslash m$. Then

$$
\sum_{k \in a \backslash m} \sum_{n \in J_{n_{k}}} \rho_{n}^{\prime}\left|\sin \lambda_{n} n \pi x\right| \leq \sum_{k \in a \backslash m} \sum_{n \in J_{n_{k}}} 3 \rho_{n}^{\prime} \delta_{n}<\infty .
$$

If $x \in E$, then

$$
\sum_{k \in a} \sum_{n \in J_{n_{k}}} \rho_{n}^{\prime}\left|\sin \lambda_{n} n \pi x\right| \leq \sum_{k \in a} \sum_{n \in J_{n_{k}}} \rho_{n}^{\prime} \lambda_{n}|\sin n \pi x| \leq \sum_{n=1}^{\infty} \rho_{n}|\sin n \pi x|<\infty .
$$

\section{The Proof of the Cases $p \mathcal{D}, \mathcal{N}_{0}, \mathcal{A}$}

All these proofs are the same and we will outline the case of $N_{0}$-sets. Let $E=\left\{x: \sum_{k=0}^{\infty}\left|\sin m_{k} \pi x\right|<\infty\right\}$ with $\left\{m_{k}\right\}_{k=0}^{\infty}$ strictly increasing. Let $\delta_{n}=1 / n^{2}$ and $\varepsilon=\delta_{n} / m_{k}$ for $k=\sum_{j=1}^{n}\left(1 / \delta_{j}\right)^{j^{2}}$. The particular steps of the proof are analogous to the case of $N$-sets.

Lemma 3.1 There is a system of functions $f_{\alpha} \in{ }^{\omega} \mathbb{R}$ for $\alpha<\mathfrak{b}$ such that

(1) $\left(\forall^{\infty} n\right) 0 \leq f_{\alpha}(n) \leq\left(1 / \delta_{n}\right)^{n^{2}} \delta_{n}^{n}$,

(2) $(\forall k \in \omega) \lim _{n \rightarrow \infty} f_{\alpha}(n) \delta_{n}^{k n}=\infty$,

(3) If $\beta<\alpha<\mathfrak{b}$, then $\left(\forall^{\infty} n\right) f_{\alpha}(n) \leq f_{\beta}(n) \delta_{n}^{n}$.

Proof. Same as the proof of Lemma 2.2.

Again it is enough to prove the following proposition. (Notice that the same proposition holds for $p D$-sets and $A$-sets. It is enough to consider either quasinormal or pointwise convergence in the definition of the set $E$ instead of absolute convergence of a series.)

Proposition 3.2 Let $\left\{n_{k}\right\}_{k=0}^{\infty}$ be a given increasing sequence of integers and $\nu<\mathfrak{t}$. If $\left\{\mathcal{I}_{n}^{\alpha}\right\}_{n=1}^{\infty}, \alpha \leq \nu$, are sequences of finite families of intervals such that $\left|\mathcal{I}_{n}\right| \leq n$ and $\left(\forall I \in \mathcal{I}_{n}\right)|I| \leq \varepsilon_{n}$, then $E \cup \bigcup_{\alpha \leq \nu} \bigcup_{m} \bigcap_{k \geq m} \cup \mathcal{I}_{n_{k}}^{\alpha}$ is an $N_{0}$-set.

ProOF. Let $\mathcal{I}_{n}^{\alpha}=\left\{\left[a_{n, l}^{\alpha}, a_{n, l}^{\alpha}+\varepsilon_{n}\right]\right\}_{l=1}^{n}$ and put

$$
P_{\alpha}=\bigcup_{m} \bigcap_{k \geq m} \bigcup \mathcal{I}_{n_{k}}^{\alpha} .
$$

In the same way as in the proof of Proposition 2.4 we build $\left\{\varphi_{\alpha}: \alpha \leq \nu\right\}$ and $\left\{a_{\alpha}: \alpha \leq \nu\right\} \subseteq[\omega]^{\omega}$ by induction so that 
(1) $\varphi_{\alpha}:\left\{n_{k}: k \in a_{\alpha}\right\} \rightarrow[\omega]^{<\omega}$

(2) $\left(\forall n \in \operatorname{dom}\left(\varphi_{\alpha}\right)\right)\left(\forall i, j \in \varphi_{\alpha}(n)\right)(\forall l=1, \ldots, n)\left|\sin \left(m_{i}-m_{j}\right) \pi a_{n, l}^{\alpha}\right|<$ $2 \pi \delta_{n}$

(3) $\left(\forall n \in \operatorname{dom}\left(\varphi_{\alpha}\right)\right)\left(\forall i \in \varphi_{\alpha}(n)\right) \sum_{j=1}^{n-1}\left(1 / \delta_{j}\right)^{j^{2}} \leq i<\sum_{j=1}^{n}\left(1 / \delta_{j}\right)^{j^{2}}$,

(4) $\left(\forall^{\infty} n \in \operatorname{dom}\left(\varphi_{\alpha}\right)\right)\left|\varphi_{\alpha}(n)\right| \geq f_{\alpha}(n)$,

(5) If $\beta \leq \alpha$, then $a_{\alpha} \subseteq^{*} a_{\beta}$ and $\left(\forall^{\infty} n \in \operatorname{dom}\left(\varphi_{\alpha}\right)\right) \varphi_{\alpha}(n) \subseteq \varphi_{\beta}(n)$.

For $\alpha \leq \nu$ limit let $U_{n}$ be the set of all pairs $(n, F)$ such that $F \in[\omega]^{<\omega}$, $|F| \geq f_{\alpha}(n),(\forall i \in F) \sum_{j=1}^{n-1}\left(1 / \delta_{j}\right)^{j^{2}} \leq j<\sum_{j=1}^{n}\left(1 / \delta_{j}\right)^{j^{2}}$, and $(\forall i, j \in$ $F)(\forall l=1, \ldots, n)\left|\sin \left(m_{i}-m_{j}\right) \pi a_{n, l}^{\alpha}\right|<2 \pi \delta_{n}$. The set $U=\bigcup_{n \in \omega} U_{n}$ is countably infinite and for $\beta<\alpha$ the sets $X_{\beta}=\left\{(n, F): F \subseteq \varphi_{\beta}(n)\right\}$ are infinite and decreasing with respect to $\subseteq^{*}$. There is an infinite set $X \subseteq U$ such that $X \subseteq^{*} X_{\beta}$ for all $\beta<\alpha$ and $\left|X \cap U_{n}\right| \leq 1$ for each $n$. Let us set $\varphi_{\alpha}=X$ and $a_{\alpha}=\left\{k: U_{n_{k}} \cap X \neq \emptyset\right\}$.

Let $\varphi=\varphi_{\nu}$ and let $a$ be the set of all $k \in a_{\nu}$ for which there are two different members $i_{k}, j_{k} \in \varphi\left(n_{k}\right), j_{k}<i_{k}$, and for each such $k$ let us set $\lambda_{k}=m_{i_{k}}-m_{j_{k}}$. Notice that both sequences $\left\{i_{k}\right\}_{k=0}^{\infty}$ and $\left\{j_{k}\right\}_{k=0}^{\infty}$ are strictly increasing. Given $\alpha \leq \nu$ for all but finitely many $k \in a, n_{k} \in \operatorname{dom}\left(\varphi_{\alpha}\right)$. Hence for all but finitely many $k \in a$

$$
\left|\sin \lambda_{k} \pi a_{n_{k}, l}^{\alpha}\right| \leq 2 \pi \delta_{n_{k}} \quad \text { for } l=1, \ldots, n_{k}
$$

and

$$
\left|\sin \lambda_{k} \pi \varepsilon_{n_{k}}\right| \leq\left(m_{i_{k}}-m_{j_{k}}\right) \pi \varepsilon_{n_{k}} \leq m_{i_{k}} \pi \varepsilon_{n_{k}} \leq \pi \delta_{n_{k}} .
$$

Consequently

$$
\left|\sin \lambda_{k} \pi x\right| \leq 3 \pi \delta_{n_{k}} \quad \text { for } x \in \bigcup \mathcal{I}_{n_{k}}^{\alpha}
$$

for all but finitely many $k \in a$. We prove that $\sum_{k \in a}\left|\sin \lambda_{k} \pi x\right|$ converges for $x \in E \cup \bigcup_{\alpha \leq \nu} P_{\alpha}$.

Let $x \in P_{\alpha}$. There is $m$ such that $x \in \bigcup \mathcal{I}_{n_{k}}^{\alpha}$ for all $k \geq m$ and such that (3.1) holds for all $k \in a \backslash m$. Then $\sum_{k \in a \backslash m}\left|\sin \lambda_{k} \pi x\right| \leq \sum_{k \in a \backslash m} 3 \pi \delta_{n_{k}}<$ $\infty$. For $x \in E, \sum_{k \in a}\left|\sin \lambda_{k} \pi x\right| \leq \sum_{k \in a}\left|\sin m_{i_{k}} \pi x\right|+\sum_{k \in a}\left|\sin m_{j_{k}} \pi x\right|$ $<\infty$.

\section{References}

[1] T. Bartoszyński and M. Scheepers, Remarks on sets related to trigonometric series, Topology Appl., 64 (1995), 133-140. 
[2] L. Bukovský, N. N. Kholshchevnikova and M. Repický, Thin sets of harmonic analysis and infinite combinatorics, Real Analysis Exchange, 20 (1994-95), 454-509.

[3] M. Repický, A family of permitted trigonometric thin sets, Proc. Amer. Math. Soc., to appear. 